\title{
Influence of Electrode Material on Space Charge Development in XLPE Plates at HVDC
}

\author{
Carl-Olof Olsson and Maria Lundmark \\ $A B B A B$, Corporate Research, Västerås, Sweden
}

\begin{abstract}
The PEA method has been used to study the evolution of electrode interface charge and space charge in $2 \mathrm{~mm}$ thick XLPE samples at $\pm 40 \mathrm{kV}$ and room temperature. The voltage polarity is switched and the transition to a steady-state at each polarity is followed. The samples are intended to be symmetric except for the electrical boundary conditions, however the measured space charge distribution is more or less unsymmetrical for all cases studied. Gold leaf, aluminium foil and graphite paint have been used as electrode materials, and the obtained results are different for the three electrode materials. Measurements with gold electrodes on three samples having different storage conditions prior to the measurements also provided significant differences between the samples.
\end{abstract}

\section{Introduction}

The pulsed electro acoustic method, PEA, is a tool to study how charge at the electrode interfaces and space charge inside insulation samples develop with time. Often a DC voltage is applied and PEA scans can be generated with and without the voltage on. The information is received as a pressure signal due to a voltage pulse creating mechanical forces where net charge is present. Good acoustic transmission is required and reflections have to be minimized on order to facilitate the interpretation. Close to the electrode interfaces there will always be interference between the pressure signals due to the interface charge, if any, and the space charge close to the electrode, if any. The interference limits the useful information that can be obtained from the charge distribution close to the interface as well as the magnitude of the interface charge. Making measurements close in time with and without voltage on, more information can be obtained since the interface charge is changing immediately when the voltage is switched off whereas the space charge is slowly decaying. The decay time is characterized by the dielectric time constant that is defined as the ratio of permittivity to conductivity. In a good insulation material the conductivity is very low, and the dielectric time constant at room temperature can be of order hours, days, or weeks. As the electric field is decreasing, the conductivity would also be decreasing leading to that charge can remain for very long time after the voltage is switched off.

The conductivity of XLPE insulation can be a complicated function of not only electric field and temperature but also of the detailed chemical composition of the sample. When peroxide is used as crosslinking agent, a number of by-products are formed. It is normally seen that the conductivity is proportional to the concentration of by-products [1-3]. Concentration gradients will cause conductivity gradients, so when DC voltage is applied the electric field will become nonuniform, and space charge will appear due to the nonuniform polarization.

In this study, the insulation samples are intended to have uniform distribution of by-products, and care is taken to prevent concentration gradients to arise due to diffusion. In this situation, it is possible to study if any other parameters or processes cause the electric field to become non-uniform. Using metallic electrodes, the diffusion of by-products is stopped at the interfaces, but this makes the insulation system different from the situation in extruded cables where semiconducting polymer layers are used as electrodes. It is thus not evident that results from these plate samples are representative if the same insulation material is used in a cable.

The influence of electrode materials for flat polyethylene samples with DC voltage has been investigated using PEA measurements [4-5] as well as leakage current measurements [6]. However, there seems to be no statistically significant conclusions drawn, so more attention to repeated measurements and improvements in the sample handling seems to be needed.

\section{Experimental arrangement}

The PEA setup consists of a cell for the test sample and the PVDF pressure sensor, a high voltage DC power supply, a pulse generator, an oscilloscope, a computer with control software and various protection devices. The voltage is $\pm 40 \mathrm{kV}$ and the pulse voltage is $7 \mathrm{kV}$. The polarity of the pulse voltage is the same as the polarity of the applied voltage. Scans without voltage are taken at every change in the applied voltage polarity. The time between scans is extended as the measurement proceeds, from five minutes up to six hours. The three samples with different electrode materials are subjected to a test sequence typically consisting of $24 \mathrm{~h}$ at $+40 \mathrm{kV}$ followed by $24 \mathrm{~h}$ at $-40 \mathrm{kV}, 24 \mathrm{~h}$ at $+40 \mathrm{kV}, 96 \mathrm{~h}$ at -40 $\mathrm{kV}$ and at least $96 \mathrm{~h}$ at $+40 \mathrm{kV}$. The three samples with different storage conditions having gold electrodes were subjected to first one week at $-40 \mathrm{kV}$ followed by at least one week at $+40 \mathrm{kV}$. The polarity of the first applied voltage was thus different for the samples with 
different electrode materials and for the samples with different storage conditions. This should provide the possibility to see if the long time evolution depends on the initial direction of the electric field.

A test sample has $210 \mathrm{~mm}$ diameter and $2 \mathrm{~mm}$ thickness. The crosslinking is made in a hot press machine at $180^{\circ} \mathrm{C}$. At the high voltage side, a $50 \mathrm{~mm}$ diameter and $1 \mathrm{~mm}$ thick XLPE disc wrapped in aluminium foil is used as electrode in order to minimize any acoustic reflections. Gold leaf of 22 carat is applied in contact with the XLPE at the centre on each side of the sample and aluminium foil is present between the gold layer and the solid electrodes. When aluminium is used as electrode material, the gold leaf is not applied. When graphite paint is the electrode material, the aluminium foil is sprayed with two layers of graphite paint. The paint is left for drying during one day before the electrodes are applied to the sample in the PEA cell.

The three samples having different electrode materials were all moulded and installed in the PEA cell the same day without any intermediate storage. The three samples for reproducibility test had different storage histories. One sample was installed in the PEA cell the same day as it was moulded. Another sample was stored in a diffusion tight wrapping for six weeks at room temperature, and the third sample was kept in an oven at $100^{\circ} \mathrm{C}$ for one week. This sample had been used earlier with gold electrodes, and the gold as well as an aluminium foil wrapping were tightly attached to the sample during the heat treatment to prevent any gradients in by-products to arise.

\section{Results and discussion}

The data from the PEA measurements are presented as the time evolution of interface electric field ratios and as individual scans of the pressure signal for selected times during the measurements, see Figs. 1 to 12 . The results for the sample with gold electrodes are shown in Figs. 1 to 4 . The measurement sequence started at $+40 \mathrm{kV}$ for $24 \mathrm{~h}$, followed by $-40 \mathrm{kV}$ for $24 \mathrm{~h},+40 \mathrm{kV}$ for $24 \mathrm{~h},-40 \mathrm{kV}$ for $96 \mathrm{~h}$ and finally $+40 \mathrm{kV}$ for $150 \mathrm{~h}$. There is a transition during the first hours after switching on the voltage as well as after each change in polarity. After the transition, there is a rather steadystate behaviour, even though a very slow change can be intimated during the $300 \mathrm{~h}$ measurement time. The interface electric field ratios at steady-state are approximately 1.4 and 0.7 for the ground electrode and high voltage electrode, respectively, when a positive voltage is applied. At negative voltage the corresponding ratios are close to unity. The space charge within the sample is characterized by zero charge in the middle of the sample and a slight gradient extending through the sample. Close to the electrodes the space charge depends on the polarity at the electrodes. At the last measurement time, there is heterocharge close to the ground electrode, but close to the high voltage electrode there is a small region with homocharge and a short distance further away from the electrode the space charge has changed polarity.

The results for the sample having plain aluminium foil as electrodes are shown in Figs. 5 to 8 . The measurement sequence started at $+40 \mathrm{kV}$ for $24 \mathrm{~h}$, followed by $-40 \mathrm{kV}$ for $24 \mathrm{~h},+40 \mathrm{kV}$ for $24 \mathrm{~h},-40 \mathrm{kV}$ for $96 \mathrm{~h}, 0 \mathrm{kV}$ for $48 \mathrm{~h},+40 \mathrm{kV}$ for $360 \mathrm{~h}, 0 \mathrm{kV}$ for $24 \mathrm{~h}$, $+40 \mathrm{kV}$ for $120 \mathrm{~h}$, and finally $0 \mathrm{kV}$ for $120 \mathrm{~h}$. The interface electric field ratios are slowly developing with time, and the behaviour is different at the two electrodes depending on the polarity. It is also seen that when changing the polarity after the $48 \mathrm{~h}$ period with $0 \mathrm{kV}$, the ratios first go back to the levels of the earlier polarity and slowly changes to what appears to be a steady-state. It took about $200 \mathrm{~h}$ from the change in polarity to reach the steady-state. At the end of the measurement sequence, the highest interface electric field ratio is found at the positive electrode. This is opposite to the results for the sample having gold electrodes that had the maximum ratio at the negative electrode. The space charge within the sample is zero in the middle and heterocharge is present in a region extending approximately $0.4 \mathrm{~mm}$ from the positive electrode. This region is thus found at the ground electrode when negative voltage is applied and at the high voltage electrode when positive voltage is applied. During the last $120 \mathrm{~h}$, when the voltage was switched off, the space charge is approximately zero except for close to the high voltage electrode. The region with space charge extends approximately $0.4 \mathrm{~mm}$ away from the electrode as estimated from Fig. 8. According to Fig. 5 , there is still some charge remaining after $120 \mathrm{~h}$, and this is also confirmed by looking into the last pressure scan, though not shown here.

The results for the sample having graphite paint electrodes are shown in Figs. 9 to 12. The measurement sequence was the same as for the sample having gold electrodes with the exception that the last part at $+40 \mathrm{kV}$ was one day longer. The data acquisition was interrupted partly during the second day and markers are therefore missing in the graph, however, the voltage was applied according to the intended sequence. The general behaviour is rather similar to the sample having gold electrodes, but all changes seem to occur more slowly. At the end of the measurement sequence when $+40 \mathrm{kV}$ was applied, the highest interface electric field ratio is found at the ground electrode, i.e. at the negative electrode. As for the other samples, the space charge is zero in the middle of the sample and heterocharge seems to develop closer to the electrodes. At the end of the long period with negative polarity, the pressure signal looks rather symmetrical. However, due to the damping of the pressure pulse, the space charge magnitude is expected to be slightly larger close to the high voltage electrode as compared to the ground electrode. At the end of the long period with positive polarity, the heterocharge region close to the ground electrode is larger than at negative polarity, and at the high voltage electrode there is even a small region with homocharge close to the electrode. 


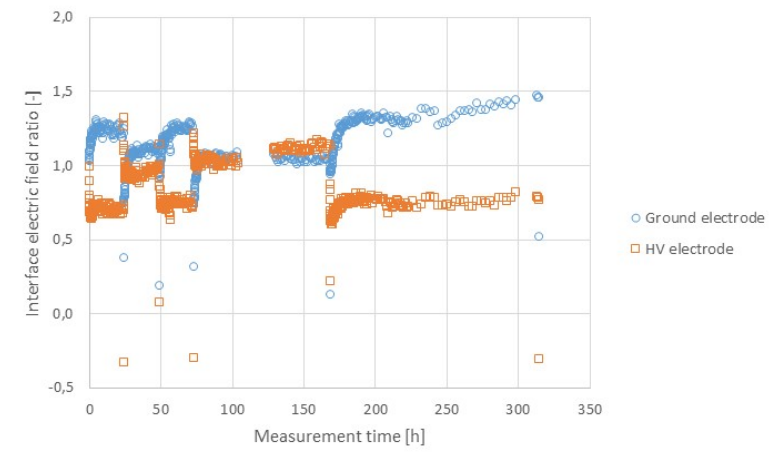

Fig. 1. Interface electric field ratios with gold electrodes.

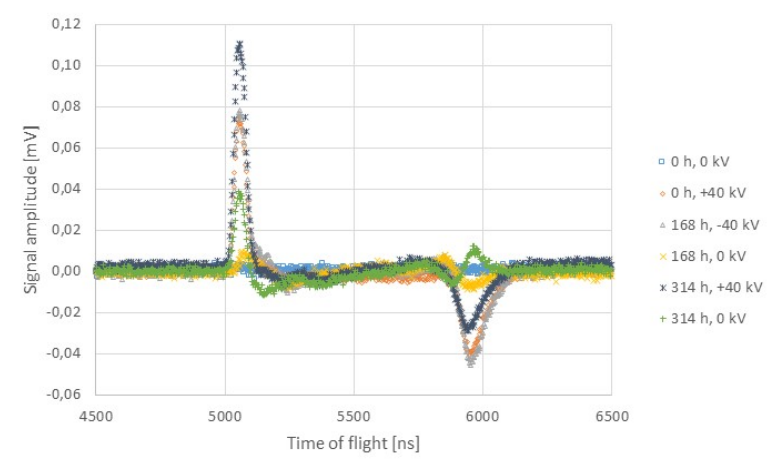

Fig. 2. Pressure signal with gold electrodes.

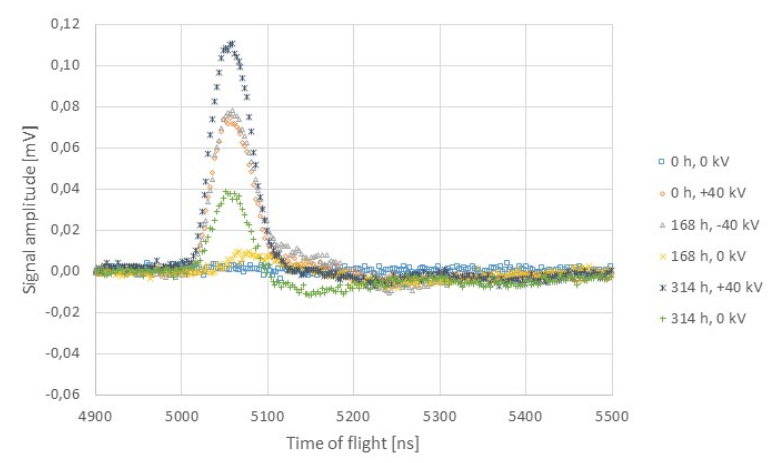

Fig. 3. Pressure signal at ground electrode with gold electrodes.

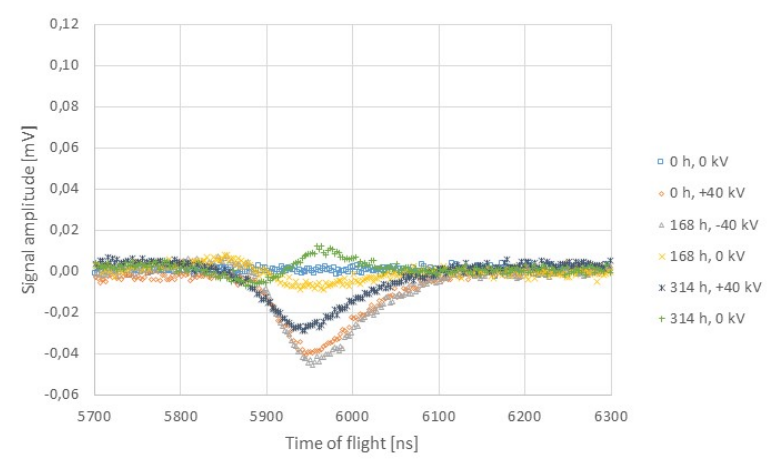

Fig. 4. Pressure signal at high voltage electrode with gold electrodes.

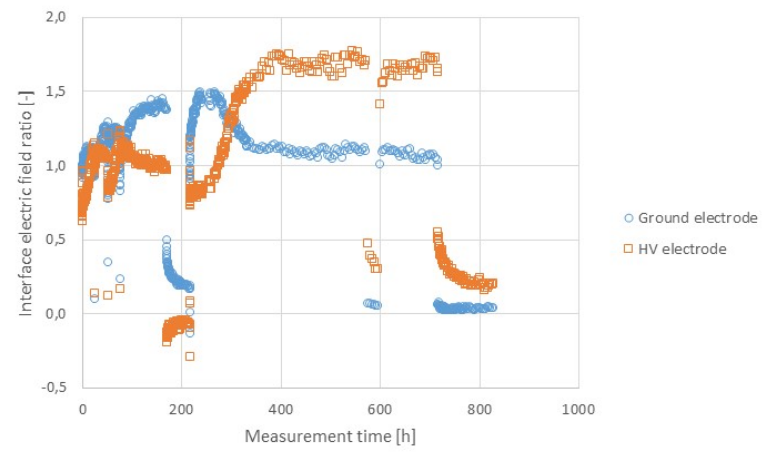

Fig. 5. Interface electric field ratios with aluminium electrodes.

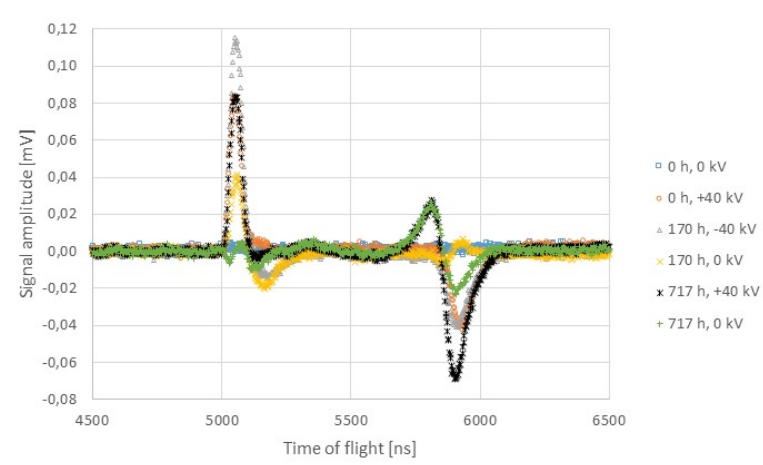

Fig. 6. Pressure signal with aluminium electrodes.

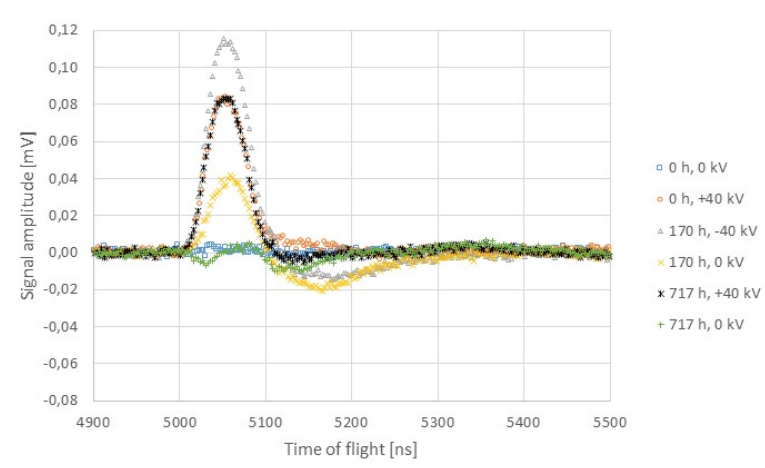

Fig. 7. Pressure signal at ground electrode with aluminium electrodes.

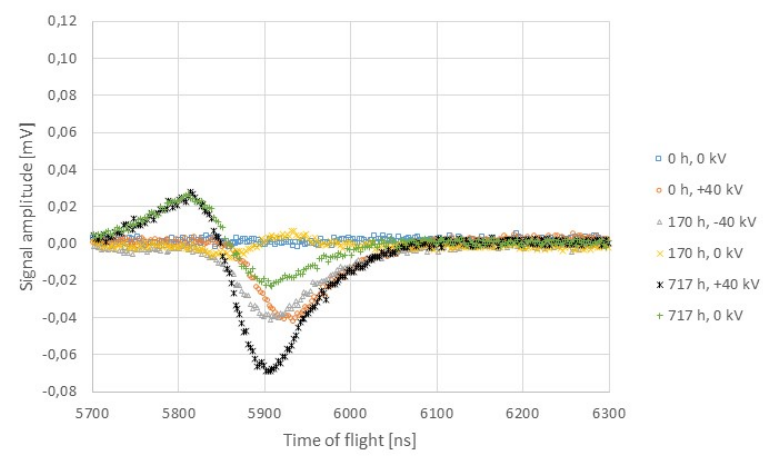

Fig. 8. Pressure signal at ground electrode with aluminium electrodes. 


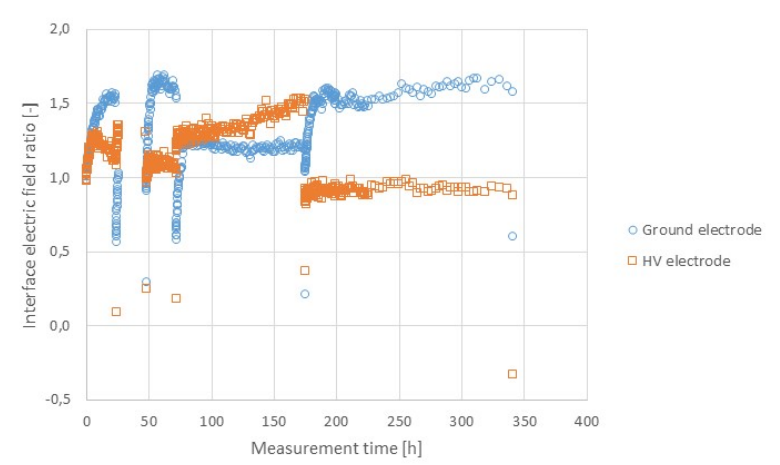

Fig. 9. Interface electric field ratios with graphite paint electrodes.

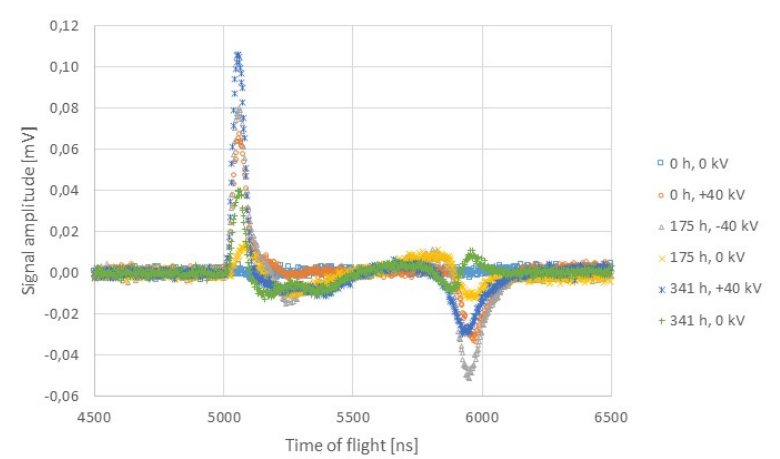

Fig. 10. Pressure signal with graphite electrodes.

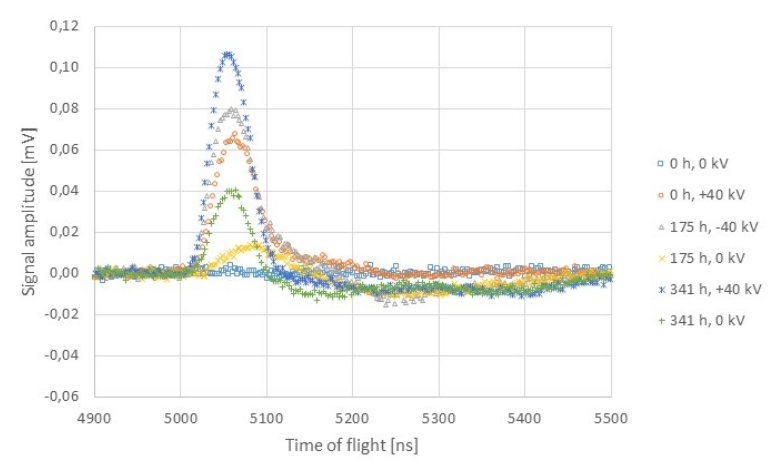

Fig. 11. Pressure signal at ground electrode with graphite paint electrodes.

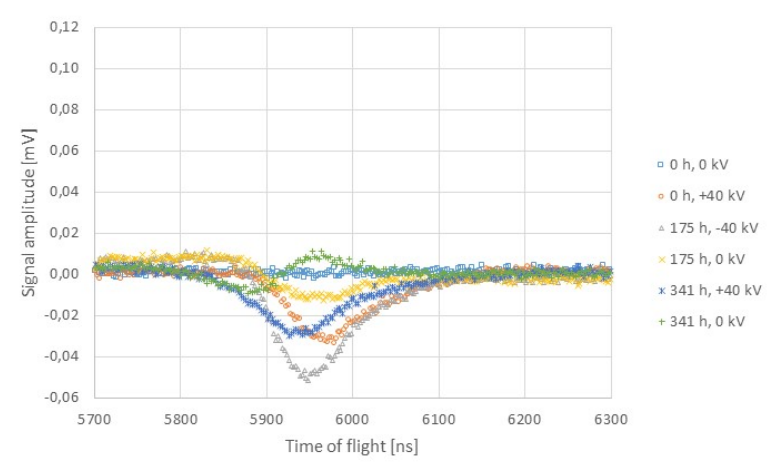

Fig. 12. Pressure signal at high voltage electrode with graphite paint electrodes.
The results from the three samples with different storage conditions all having gold electrodes are shown in Fig. 13. The evolution of the interface electric field ratios as function of time are shown for one week at negative polarity followed by one to three weeks at positive polarity. Large variations are seen between the samples. The transition to a steady-state takes different times; the shorter time for the sample stored at room temperature and the longer time for the fresh sample. The maximum interface electric field ratio is also different. At the end of the first week with negative polarity, the highest ratio is found for the ground electrode of the heated sample followed by the high voltage electrode of the fresh sample. After the subsequent week at positive polarity, the highest ratio is found at the ground electrode of the fresh sample followed by the high voltage electrode of the heated sample. It seems thus as the electric field distribution within each sample has been mirrored due to the polarity switch. However, the maximum field is found at the negative electrodes for the fresh sample whereas it is found at the positive electrodes for the heated sample.

Comparing Fig. 1 and Fig. 13, it can be seen that the behaviour of the sample of Fig. 1 with gold electrodes get the highest interface electric field ratio at the ground electrode when a positive voltage is applied, whereas the same sample after heat treatment of Fig. 13 shows the highest ratio at the ground electrode when a negative voltage is applied. It can however not be revealed if the difference is due to the heat treatment or if it is due to the polarity of the first voltage during the test sequences. Comparing the fresh sample of Fig. 13 with the, also, fresh sample in Fig. 1 it is seen that in both cases the highest ratio is found at the negative electrode, i.e. at the ground electrode of Fig. 1 and at the high voltage electrode of Fig. 13.

In Figs. 14 and 15, pressure signals are shown for the three samples after $-40 \mathrm{kV}$ has been applied during one week. The voltage was on when the signals were recorded for Fig. 14 and switched off for Fig. 15. All the curves are close to zero in the middle of the sample, and they indicate positive charge towards the high voltage electrode and negative charge towards the ground electrode. In the fresh sample the positive charge is dominating, whereas in the sample stored at room temperature the charge distribution seems to be more symmetrical. In the sample heat treated at $100^{\circ} \mathrm{C}$, there is a weak gradient in charge across the sample, and close to the ground electrode there is a small region with rather large negative charge.

It can be noted in Fig. 15 that the peaks due to the electrode interface charge appears to move slightly when comparing the three samples at the ground electrode. This is an effect of the interference of the pressure pulse generated at the interface charge and the pressure pulse generated due to the space charge in the insulation close to the interface. The heated sample provides the highest positive peak, but it also has a negative peak adjacent to the interface that will interfere 
with at least the right hand flank of the interface peak. The stored sample also has a

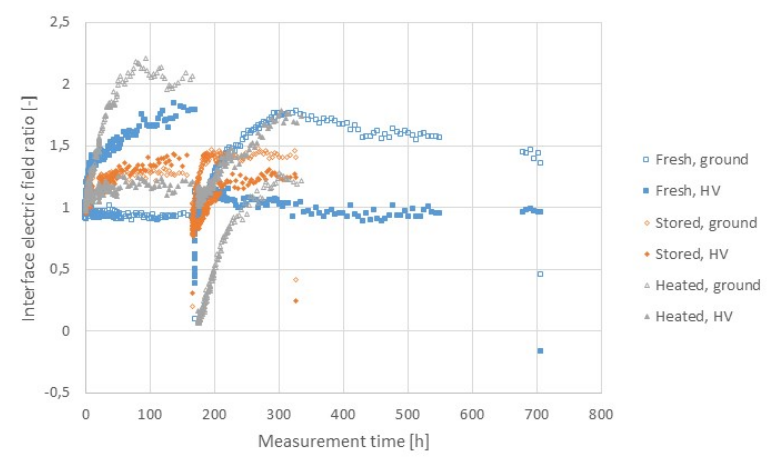

Fig. 13. Three samples with different storage conditions having gold electrodes. Interface electric field ratios. One week at negative polarity followed by one to three weeks with positive polarity.

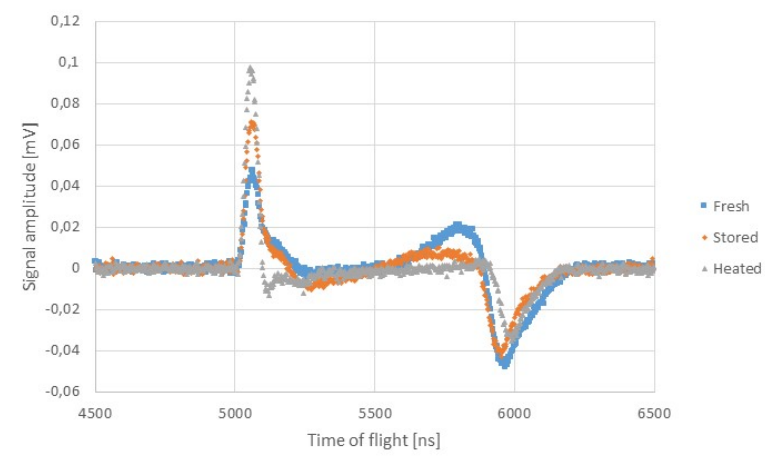

Fig. 14. Pressure signals after one week at $-40 \mathrm{kV}$. The voltage was on when the signals were recorded. Ground electrode at the left hand peaks and high voltage electrode at the right hand peaks.

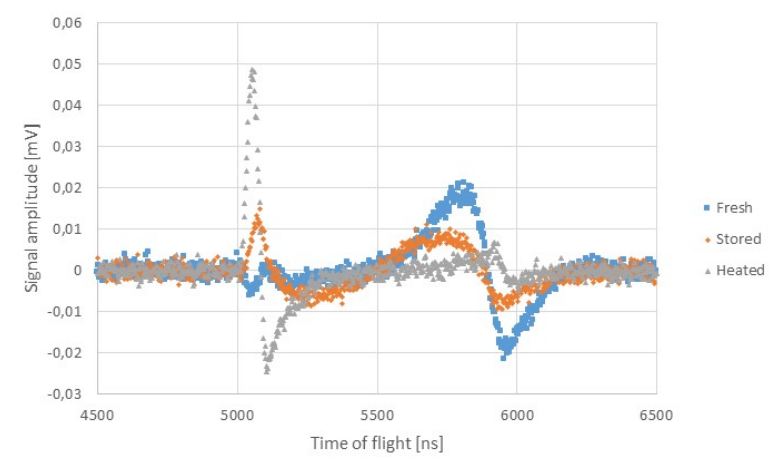

Fig. 15. Pressure signals after one week at $-40 \mathrm{kV}$. The voltage was off when the signals were recorded. Ground electrode at the left hand peaks and high voltage electrode at the right hand peaks.

positive peak, and this peak location coincides with the same peak in the scan with voltage on.

The fresh sample has rather large positive space charge and the interface charge is therefore negative at both interfaces. The weak peak at the ground electrode is slightly displaced to the left that might be due to the small positive charge adjacent to the electrode. Similar observations can be made by looking at the pressure scans from the three samples having different electrode materials. The slight difference in positions of the interface peaks at the high voltage electrode is mainly due to the different thickness of the samples. The stored sample has the smallest thickness followed by the fresh sample, and the heated sample has the largest thickness. These thickness variations are most likely due to the storage conditions that would influence the relaxation of mechanical stresses created during the moulding sequence. There is also a small variation in the original thickness of fresh moulded samples.

The data shown in Fig. 13 are obtained from a large number of scans similar to those shown in Figs. 14 and 15 , and it is evident that great care need to be taken when deciding which data points in the scans actually represent the values for the interface peaks. As an example, the interface peak at the ground electrode for the heated sample after the polarity switch is almost zero when the positive voltage is switched on. However, just next to the interface location there is a significant peak due to space charge. The reading representative for the interface charge must thus be taken at exactly the correct position and not be confused by the adjacent peak. No filtering has been applied to the pressure signals since this could cause additional difficulties in the interpretation of the data.

\section{Concluding remarks}

Measurement times of one day, one week, and sometimes longer have been used to follow the development of interface charge and space charge in flat XLPE samples at $20 \mathrm{kV} / \mathrm{mm}$ average electric field and at room temperature. Diffusion of peroxide by-products away from the samples during the measurements has been prevented by using metallic electrodes.

The results are rather different depending on if gold, aluminium, or graphite paint is used at the electrodes.

Three similar samples with gold electrodes but with different storage conditions before the measurements were started show also rather different results.

When switching on the voltage as well as when the voltage polarity is switched, there is a transition time of less than one day to reach a quasi-steady-state. This transition time is in line with the dielectric time constant that is estimated to be of the order of 1 to $10 \mathrm{~h}$ knowing that the average conductivity is of the order of $10^{-15}$ $\mathrm{S} / \mathrm{m}$. Following the evolution during longer time, it is seen that there are also other changes taking place that have longer time constants.

In order to draw any conclusions from these measurement results, it seems as the samples need to be characterized with respect to more parameters than the electrode materials and the distribution of peroxide byproducts. 


\section{References}

[1] K. Ogawa, T. Suzuki, S. Katakai, M. Kanaoka, and Y. Sekii, "DC characteristics of cable insulating materials”, Proc. $21^{\text {st }}$ Symp. Elec. Ins. Mat., 1988, pp. 275-278.

[2] C. O. Olsson and M. Jeroense, "Finite element simulations of DC electric field development in polymer insulation”, NORDIS, Göteborg, Sweden, 2009.

[3] C. O. Olsson, B. Källstrand, B. Ma, M. Lundmark, K. Johansson, S. Arnsten, A. Abbasi, M. Saltzer, M. Jeroense, "Space charge distribution in XLPE plates with non-uniform conductivity", Jicable, Versailles, France, 2015.

[4] G. Chen, T. Y. G. Tay, A. E. Davies, Y. Tanaka, and T. Takada, "Electrodes and charge injection in low-density polyethylene using the pulsed electroacoustic technique”, IEEE Trans. Dielec. Electr. Ins., Vol. 8, 2001, pp. 867-873.

[5] L. Li, B. Han, W. Song, X. Wang, and Q. Q. Lei, "The effect of the semiconductive screen on space charge suppression in cross-linked polyethylene”, Chin. Phys. Lett., Vol. 31, 2014, paper 107301.

[6] J. Viertel, L. Petersson, A. Friberg, G. Dominguez, and C. Törnkvist, "Electrode influence on DC conductivity measurements of low density poly ethylene”, ICSD, Bologna, Italy, 2013. 